\title{
Adiponectin and Leptin Metabolic Biomarkers in Chinese Children and Adolescents
}

\author{
Jie Mi, ${ }^{1}$ Mercedes Nancy Munkonda, ${ }^{2}$ Ming Li, ${ }^{3}$ Mei-Xian Zhang, ${ }^{1}$ Xiao-Yuan Zhao, \\ Ponce Cedric Wamba Fouejeu, ${ }^{4}$ and Katherine Cianflone ${ }^{2}$ \\ ${ }^{1}$ Department of Epidemiology, Capital Institute of Pediatrics, Beijing 100020, China \\ ${ }^{2}$ Department of Endocrinology, Peking Union Medical College Hospital, Peking Union Medical College and Chinese Academy of \\ Medical Sciences, Beijing 100730, China \\ ${ }^{3}$ Centre de Recherche Institut, Universitaire de Cardiologie et Pneumologie de Quebec, Université Laval, 2725 Chemin Ste-Foy, \\ Québec, QC, Canada G1V 4 G5 \\ ${ }^{4}$ Laboratory of Nutrition and Nutritional Biochemistry, University of Yaoundé, Yaoundé, Cameroon
}

Correspondence should be addressed to Katherine Cianflone, katherine.cianflone@crhl.ulaval.ca

Received 25 June 2010; Accepted 6 September 2010

Academic Editor: Paul Trayhurn

Copyright (C) 2010 Jie Mi et al. This is an open access article distributed under the Creative Commons Attribution License, which permits unrestricted use, distribution, and reproduction in any medium, provided the original work is properly cited.

\begin{abstract}
Objective. To evaluate leptin and adiponectin as biomarkers of metabolic syndrome (MS) risk factors even in nonobese children/adolescents. Methods. Serum leptin, adiponectin, leptin:adiponectin ratio, lipids, glucose, and insulin concentrations as well as body size parameters and pubertal development were evaluated in a large population of Chinese children/adolescents ( $n=3505,6-18$ years, 1722 girls and 1783 boys). Results. Leptin concentration increased while adiponectin decreased with obesity, both were influenced by pubertal development. Central obesity had an additive effect on leptin levels (above obesity alone). Leptin/adiponectin increased 8.4-fold and 3.2-fold in overweight/obesity, and 15.8- and 4.5-fold with obesity plus MS, in early and late puberty, respectively. Even in normal weight children/adolescents, higher leptin and lower adiponectin concentrations associated with increased risk profile. Conversely, overweight/obese with lower leptin or higher adiponectin concentrations had a less compromised metabolic profile. Conclusion. Leptin, adiponectin, and leptin:adiponectin ratio are informative biomarkers for obesity, central obesity, MS, and abnormal metabolic profile even in normal weight children/adolescents.
\end{abstract}

\section{Introduction}

The alarming increase in obesity worldwide is of concern, due to the associations of obesity with metabolic syndrome (MS), insulin resistance, Type 2 diabetes, dyslipidaemia and cardiovascular disease $[1,2]$. This increase is noted even in populations previously at reduced risk, such as Asia [3], even in children [4]. MS is characterized by central obesity, insulin resistance, hyperglycaemia, dyslipidaemia (increased triglyceride and decreased HDL), and hypertension; all risk factors for cardiovascular disease and Type 2 diabetes mellitus. Characterization of MS potentially identifies individuals predisposed to cardiovascular disease and Type 2 diabetes, allowing measures to be instituted early-on [3]. Adult urban versus rural populations are at higher MS risk [5] and prevalence varies with gender, age, ethnic background, and residence
$[1,5]$. Puberty, with naturally occurring growth spurts and hormonal changes coupled to behavioural changes, is a critical period for development of obesity and childhood MS [6]. Further, the associated pathological processes and risk factors have been observed in obese children and adolescents [7-9].

Adipose tissue secretes adipokines influencing body weight, glucose, and lipid metabolism [7]. Adiponectin, a collagen-like protein exclusively expressed in adipose tissue, has antiatherogenic, antidiabetic and anti-inflammatory properties [10-12]. Increased adiponectin is associated with a lower risk of impaired glucose tolerance, decreased myocardial infarction risk, and has been proposed as a biomarker of early atherosclerosis $[10,11,13]$. Adiponectin decreases in obesity and related diseases, and may be involved 
TABLE 1: Anthropometric and metabolic parameters in girls and boys. Data are expressed as mean \pm SD where $* * P<.01$ and $* * * P<.001$.

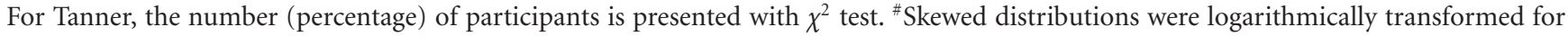
$t$-test.

\begin{tabular}{|c|c|c|c|}
\hline Variables & All & Girls & Boys \\
\hline$n$ & 3505 & 1722 & 1783 \\
\hline Age (years) & $12.4 \pm 3.1$ & $12.6 \pm 3.1^{* * *}$ & $12.2 \pm 3.0$ \\
\hline Pubertal development & 3388 & 1693 & 1695 \\
\hline Tanner stage I & $1001(29.5)$ & $336(19.8)$ & $665(39.2)$ \\
\hline Tanner stage II & $486(14.3)$ & $216(12.8)$ & $270(15.9)$ \\
\hline Tanner stage III & $464(13.7)$ & $208(12.3)$ & $256(15.1)$ \\
\hline Tanner stage IV & $813(24.0)$ & $610(36.0)$ & $203(12.0)$ \\
\hline Tanner stage V & $624(18.4)$ & $323(19.1)^{* * *}$ & $301(17.8)$ \\
\hline Leptin $(\mathrm{ng} / \mathrm{ml})^{\#}$ & $10.0 \pm 11.4$ & $11.0 \pm 11.6^{* * *}$ & $9.1 \pm 11.2$ \\
\hline Adiponectin $(\mu \mathrm{g} / \mathrm{ml})^{\#}$ & $12.8 \pm 7.4$ & $13.2 \pm 7.2^{* * *}$ & $12.3 \pm 7.6$ \\
\hline Ratio leptin/adiponectin ${ }^{\#}$ & $1.24 \pm 1.90$ & $1.33 \pm 2.10^{* * *}$ & $1.15 \pm 1.68$ \\
\hline Body mass index $\left(\mathrm{kg} / \mathrm{m}^{2}\right)$ & $21.9 \pm 4.9$ & $21.0 \pm 4.5^{* * *}$ & $22.8 \pm 5.2$ \\
\hline Waist circumference $(\mathrm{cm})$ & $72.4 \pm 13.1$ & $68.6 \pm 10.8^{* * *}$ & $76.0 \pm 14.0$ \\
\hline Waist to height ratio & $0.47 \pm 0.07$ & $0.46 \pm 0.06^{* * *}$ & $0.49 \pm 0.07$ \\
\hline Fat mass percentage (\%) & $24.4 \pm 8.5$ & $25.6 \pm 9.0^{* * *}$ & $23.2 \pm 7.8$ \\
\hline Systolic BP (mm Hg) & $107.6 \pm 13.9$ & $104.7 \pm 12.3^{* * *}$ & $110.4 \pm 14.8$ \\
\hline Diastolic BP (mm Hg) & $67.8 \pm 10.0$ & $66.8 \pm 9.4^{* * *}$ & $68.8 \pm 10.5$ \\
\hline Triglycerides $(\mathrm{mmol} / \mathrm{l})^{\#}$ & $1.03 \pm 0.56$ & $1.03 \pm 0.55$ & $1.02 \pm 0.57$ \\
\hline Total cholesterol (mmol/l) & $4.09 \pm 0.79$ & $4.11 \pm 0.81$ & $4.07 \pm 0.78$ \\
\hline $\mathrm{HDL}-\mathrm{C}(\mathrm{mmol} / \mathrm{l})$ & $1.40 \pm 0.32$ & $1.42 \pm 0.30^{* *}$ & $1.39 \pm 0.34$ \\
\hline LDL cholesterol $(\mathrm{mmol} / \mathrm{l})$ & $2.54 \pm 0.72$ & $2.56 \pm 0.74$ & $2.53 \pm 0.71$ \\
\hline Glucose $(\mathrm{mmol} / \mathrm{l})$ & $5.10 \pm 0.62$ & $5.03 \pm 0.67^{* * *}$ & $5.15 \pm 0.55$ \\
\hline Insulin $(\mathrm{mU} / \mathrm{l})^{\#}$ & $10.46 \pm 9.95$ & $10.18 \pm 8.92$ & $10.72 \pm 10.84$ \\
\hline HOMA-IR & $2.43 \pm 2.62$ & $2.33 \pm 2.29$ & $2.52 \pm 2.90$ \\
\hline
\end{tabular}

Table 2: Pearson correlation coefficients of leptin and adiponectin. \#Skewed distributions logarithmically transformed for Pearson correlation. Significances are ${ }^{*} P<.05,{ }^{* *} P<.01$, and ${ }^{* * *} P<.001$.

\begin{tabular}{|c|c|c|c|c|c|c|c|c|}
\hline \multirow{3}{*}{ Variables } & \multicolumn{4}{|c|}{ Leptin $^{\#}$} & \multicolumn{4}{|c|}{ Adiponectin $^{\#}$} \\
\hline & \multicolumn{2}{|c|}{ Girls } & \multicolumn{2}{|c|}{ Boys } & \multicolumn{2}{|c|}{ Girls } & \multicolumn{2}{|c|}{ Boys } \\
\hline & NonOwt & Owt/Ob & NonOwt & Owt/Ob & NonOwt & Owt/Ob & NonOwt & Owt/Ob \\
\hline$n$ & 963 & 722 & 680 & 1064 & 986 & 725 & 708 & 1066 \\
\hline Age & $0.517^{* * *}$ & $0.253^{* * *}$ & 0.033 & $-0.172^{* * *}$ & $-0.191^{* * *}$ & $-0.201^{* * *}$ & $-0.350^{* * *}$ & $-0.366^{* * *}$ \\
\hline Leptin $^{\#}$ & - & - & - & - & $-0.219^{* * *}$ & $-0.097^{* *}$ & $-0.114^{* *}$ & 0.018 \\
\hline Adiponectin ${ }^{\#}$ & $-0.219^{* * *}$ & $-0.097^{* *}$ & $-0.114^{* *}$ & 0.018 & - & - & - & - \\
\hline Body mass index & $0.691^{* * *}$ & $0.469^{* * *}$ & $0.377^{* * *}$ & $0.272^{* * *}$ & $-0.282^{* * *}$ & $-0.240^{* * *}$ & $-0.301^{* * *}$ & $-0.329^{* * *}$ \\
\hline Waist circumference & $0.708^{* * *}$ & $0.466^{* * *}$ & $0.351^{* * *}$ & $0.291^{* * *}$ & $-0.267^{* * *}$ & $-0.288^{* * *}$ & $-0.329^{* * *}$ & $-0.346^{* * *}$ \\
\hline Waist to height ratio & $0.375^{* * *}$ & $0.354^{* * *}$ & $0.432^{* * *}$ & $0.466^{* * *}$ & $-0.180^{* * *}$ & $-0.141^{* * *}$ & NS & $-0.082^{* *}$ \\
\hline Fat mass percentage & $0.734^{* * *}$ & $0.498^{* * *}$ & $0.446^{* * *}$ & $0.386^{* * *}$ & $-0.271^{* * *}$ & $-0.230^{* * *}$ & $-0.157^{* * *}$ & NS \\
\hline Systolic BP & $0.294^{* * *}$ & $0.150^{* * *}$ & NS & NS & $-0.173^{* * *}$ & $-0.109^{* *}$ & $-0.202^{* * *}$ & $-0.225^{* * *}$ \\
\hline Diastolic BP & $0.274^{* * *}$ & $0.133^{* * *}$ & NS & NS & $-0.108^{* * *}$ & NS & $-0.099^{* *}$ & $-0.126^{* * *}$ \\
\hline Triglycerides ${ }^{\#}$ & $0.342^{* * *}$ & $0.218^{* * *}$ & $0.281^{* * *}$ & $0.272^{* * *}$ & $-0.144^{* * *}$ & $-0.172^{* * *}$ & $-0.109^{* *}$ & $-0.144^{* * *}$ \\
\hline Total cholesterol & NS & NS & $0.140^{* * *}$ & $0.154^{* * *}$ & NS & NS & NS & NS \\
\hline HDL-C & $-0.193^{* * *}$ & NS & -0.088 & NS & $0.246^{* * *}$ & $0.231^{* * *}$ & $0.231^{* * *}$ & $0.326^{* * *}$ \\
\hline LDL cholesterol & NS & NS & $0.153^{* * *}$ & $0.153^{* * *}$ & NS & NS & NS & NS \\
\hline Glucose & $0.121^{* * *}$ & NS & NS & NS & NS & NS & NS & 0.068 \\
\hline Insulin ${ }^{\#}$ & $0.510^{* * *}$ & $0.425^{* * *}$ & $0.366^{* * *}$ & $0.339^{* * *}$ & $-0.201^{* * *}$ & $-0.211^{* * *}$ & $-0.201^{* * *}$ & $-0.270^{* * *}$ \\
\hline HOMA-IR ${ }^{\#}$ & $0.502^{* * *}$ & $0.409^{* * *}$ & $0.364^{* * *}$ & $0.326^{* * *}$ & $-0.200^{* * *}$ & $-0.197^{* * *}$ & $-0.196^{* * *}$ & $-0.247^{* * *}$ \\
\hline
\end{tabular}



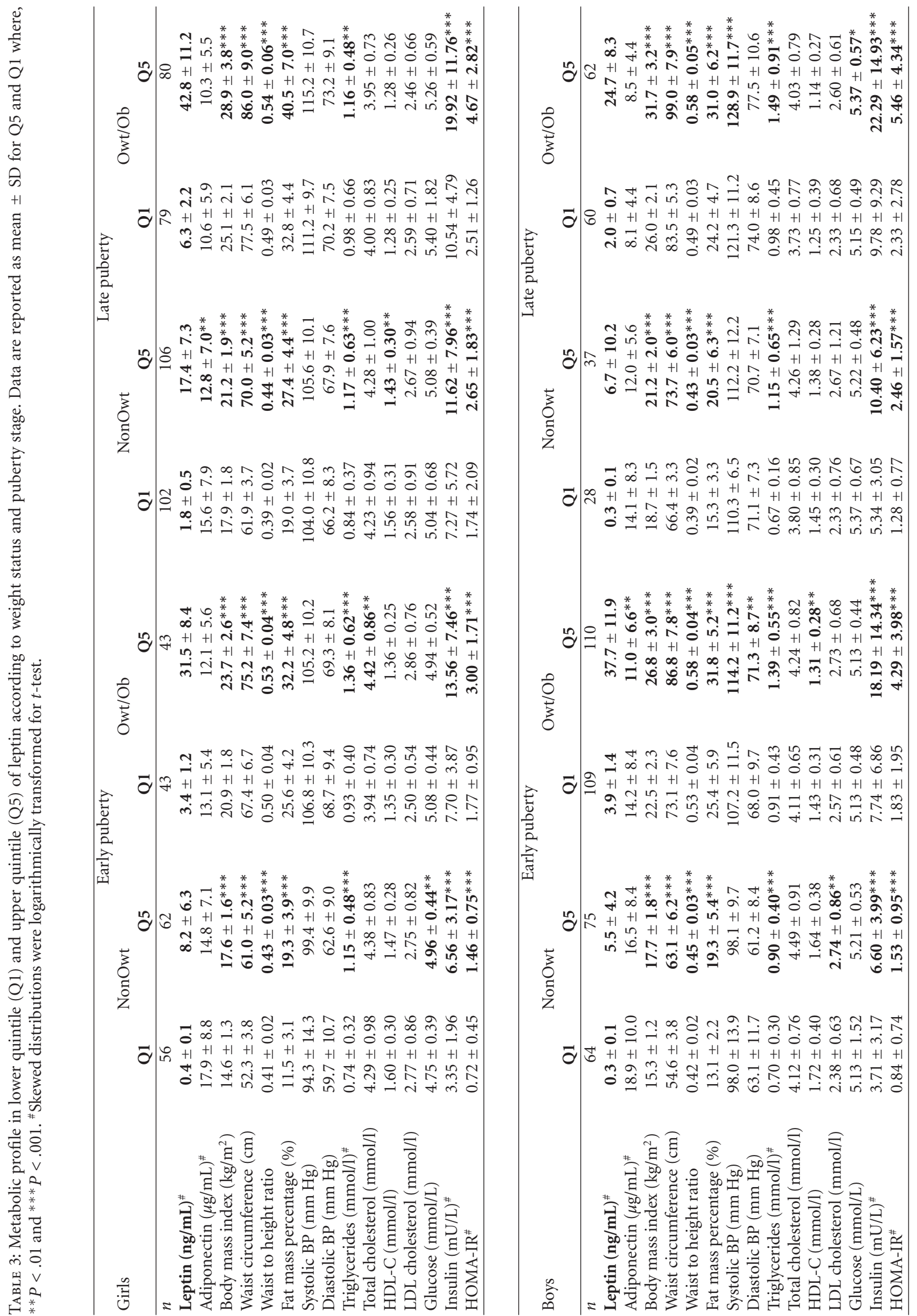

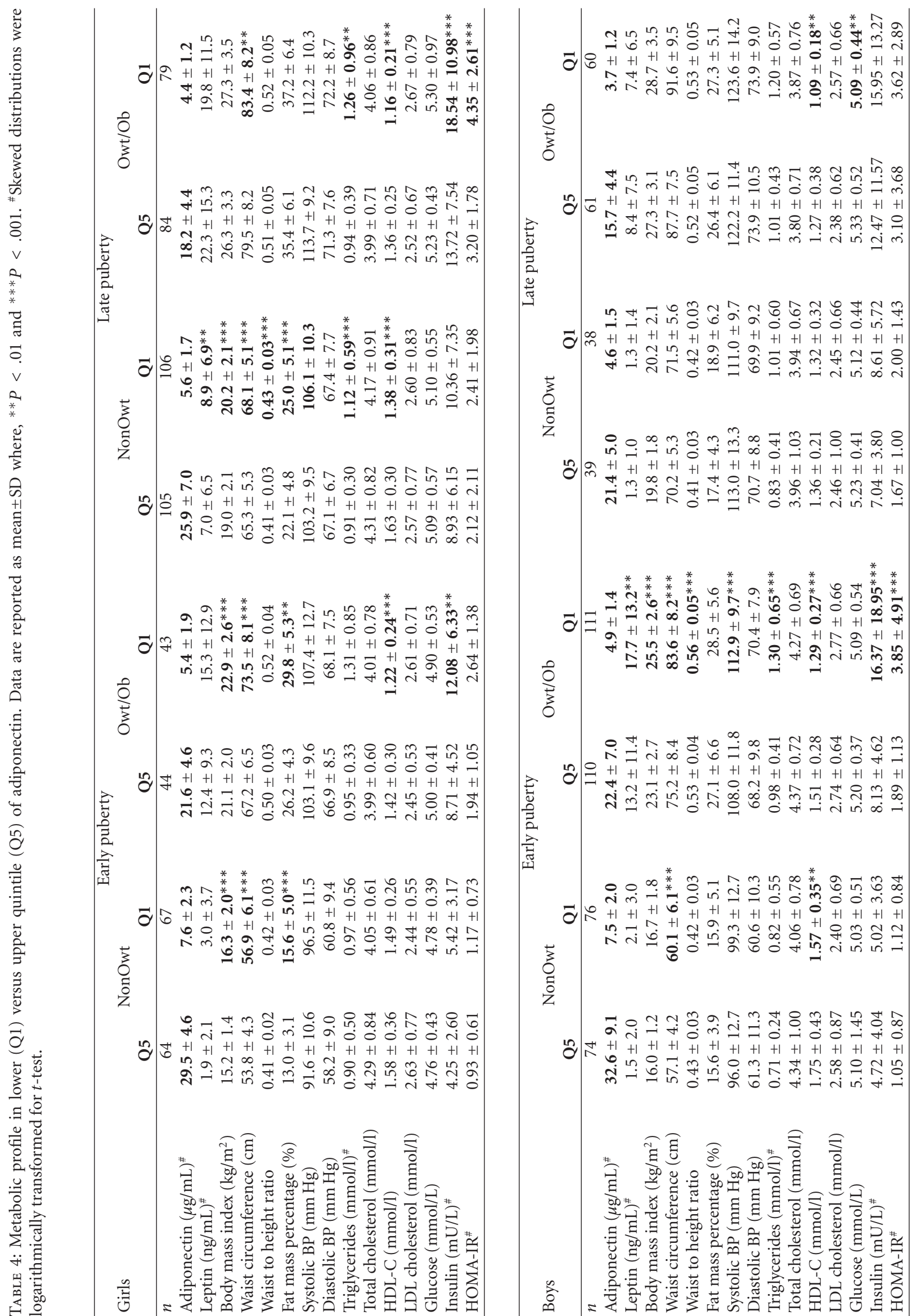
in cardiovascular disease and Type 2 diabetes mellitus pathology [14].

Leptin, a cytokine-like molecule secreted by adipose tissue, regulates adipose mass and body weight by inhibiting food intake and stimulating energy expenditure $[15,16]$. Leptin increases in obesity, Type 2 diabetes mellitus, hypertension and MS. Numerous publications in adults suggest leptin as a biomarker for obesity, insulin resistance, and MS $[15,16]$.

Obesity, MS prevalence and associated risk factors in children and adolescents have increased dramatically over decades $[4,8]$, including in Chinese populations $[1,2,17$, 18]. This relationship is difficult to evaluate as obesityinduced acute insulin resistance is usually identified later, and many changes are associated with pubertal and socioeconomic changes $[6,19,20]$. Leptin and adiponectin are proposed as biomarkers in children for predicting MS, Type 2 diabetes, or cardiovascular disease [21]; however, the lack of defined cutoff markers complicates this [22]. Many studies on leptin and/or adiponectin in children [12, 23-25], including Asian children [5, 26, 27], suffer from small sample sizes, with only two studies over 800-1000 children, limiting subgroup analysis.

Recently, leptin-to-adiponectin ratio was proposed as a biomarker with the benefits of both indices [28-30] included in Chinese adults [5]. In Hispanic youth [31] leptin-toadiponectin ratio provided an independent predictor of insulin sensitivity during growth [31].

In this study, we evaluated adipokines, anthropometric, and lipid parameters in a large study of 3505 Chinese children and adolescents aged 6-18 years old. We demonstrate that even in normal weight children and adolescents, leptin and adiponectin are informative biomarkers of risk, and also discriminate between lower versus higher risk in overweight/obese subjects.

\section{Materials/Subjects and Methods}

2.1. Subjects and Samples Collection. Subjects were recruited from a cross-sectional population-based survey: the Beijing Child and Adolescent Metabolic Syndrome (BCAMS) study as described elsewhere [32]. The cohort included 3505 children, 1722 girls, and 1783 boys of normal weight, overweight, and obese defined by age-gender-specific BMI according to the International Obesity Task Force (IOTF) [33]. Signed informed consent was obtained from participants and/or parents/guardians. The BCAMS study was approved by the Ethics Committee at Capital Institute of Pediatrics in Beijing. Subjects were evaluated for height, weight, waist circumference, Tanner stage, and fat mass \% (by bioimpedance analysis).

2.2. Clinical and Metabolic Parameters. Venous blood samples were collected by direct venipuncture after an overnight (minimum $12 \mathrm{~h}$ ) fast. The samples were centrifuged, serum and plasma aliquoted and immediately frozen and maintained at $-80^{\circ} \mathrm{C}$ for later analysis of lipids and hormones. Samples were collected and analysed over a two-year period.
Blood samples were analyzed for glucose, triglyceride (TG), total cholesterol (TC), high-density lipoprotein cholesterol (HDL-C), low-density lipoprotein cholesterol (LDL-C), insulin, adiponectin, and leptin. Serum lipids (enzymatic methods) and plasma glucose (glucose oxidase method) were assayed using the Hitachi $7060 \mathrm{C}$ automatic biochemistry analysis system. HDL-C and LDL-C were measured directly. Serum insulin was measured by monoclonal antibody-based sandwich enzyme-linked immunosorbent assays (ELISA) [34], developed in Key Laboratory of Endocrinology, Peking Union Medical College Hospital with interassay CVs of $<9.0 \%$ and no cross-reactivity to proinsulin $(<0.05 \%)$. Serum adiponectin was measured by ELISA with intra-assay and interassay coefficients of variation of $<5.4 \%$ and $<8.5 \%$, respectively, (see details elsewhere) [32]. All antibodies, reagents and adiponectin standard were purchased from Phoenix Pharmaceuticals Inc. (Belmont, CA, USA).

Serum leptin was measured by ELISA [35] using rabbit polyclonal (PAb) and mouse monoclonal (MAb) antihuman leptin antibodies prepared following injection of human leptin (R\&D System, USA). Plates coated with $3 \mu \mathrm{g} / \mathrm{mL}$ PAb in carbonate buffer were incubated overnight at $4^{\circ} \mathrm{C}$, blocked for $1 \mathrm{~h}$ with $1 \%$ BSA in phosphate-buffered saline (PBS). Standards $(50 \mu \mathrm{L}, 0-12.5 \mathrm{ng} / \mathrm{ml}, 9$ serial dilutions in $1 \%$ BSA-PBS), diluted blood samples and quality controls were loaded. Biotin-labeled MAb (50 uL, diluted 1:1000) were added and incubated 1.5 hours at $37^{\circ} \mathrm{C}$. For the following steps, plates were washed between each step: (i) $100 \mu \mathrm{L}$ avidin-HRPO (diluted $1: 1000$ ), 30 minutes $37^{\circ} \mathrm{C}$; (ii) $100 \mu \mathrm{L}$ chromogenic substrate TMB and development solution, 10 minutes $37^{\circ} \mathrm{C}$; (iii) $100 \mu \mathrm{L} 1 \mathrm{M} \mathrm{H}_{2} \mathrm{SO}_{4}$; (iv) $\mathrm{OD}$ at $450 \mathrm{~nm}$. Sensitivity was $0.03 \mathrm{ng} / \mathrm{ml}$; range $0.05-5 \mathrm{ng} / \mathrm{ml}$, intra-assay and interassay coefficients of variation were $<7.4 \%$ and $<9.3 \%$.

2.3. Classification of Pediatric Metabolic Syndrome. Pediatric metabolic syndrome was defined by the presence of three or more of the following five components [36]: (i) central obesity defined as age/gender waist circumference $\geq 90$ th percentile (established based on the BCAMS study); (ii) elevated age/gender systolic and/or diastolic blood pressure $\geq 90$ th percentile (BCAMS); (iii) hypertriglyceridemia $\geq 1.24 \mathrm{mM}$, (90th percentile reference population); (iv) low-serum HDL$\mathrm{C}<1.03 \mathrm{mM}$ (5th percentile reference population); (v) impaired fasting glucose $\geq 5.6 \mathrm{mM}$.

2.4. Calculations and Statistical Analysis. Unless otherwise stated, results are mean \pm standard deviation (SD). BMI was calculated as weight $/$ height $^{2}\left(\mathrm{~kg} / \mathrm{m}^{2}\right)$. Insulin resistance calculated by homeostasis-model-assessment-insulinresistance (HOMA-IR) is (insulin $\mathrm{IU}_{-1}^{-1}$ ) $\times$ (glucose $\left.\mathrm{mmol}^{-1}\right) / 22.5$. Analyses and graphics were performed using SysStat, SigmaStatV3.5, and GraphPadPrismV5.0. All skewed distributions were log transformed for analysis. $T$-Test was used for comparison of two groups. One-way or twoway ANOVA was used for comparison of multiple groups (as indicated) with Tukey-Kramer post hoc comparison being used to evaluate group differences. Where indicated, 


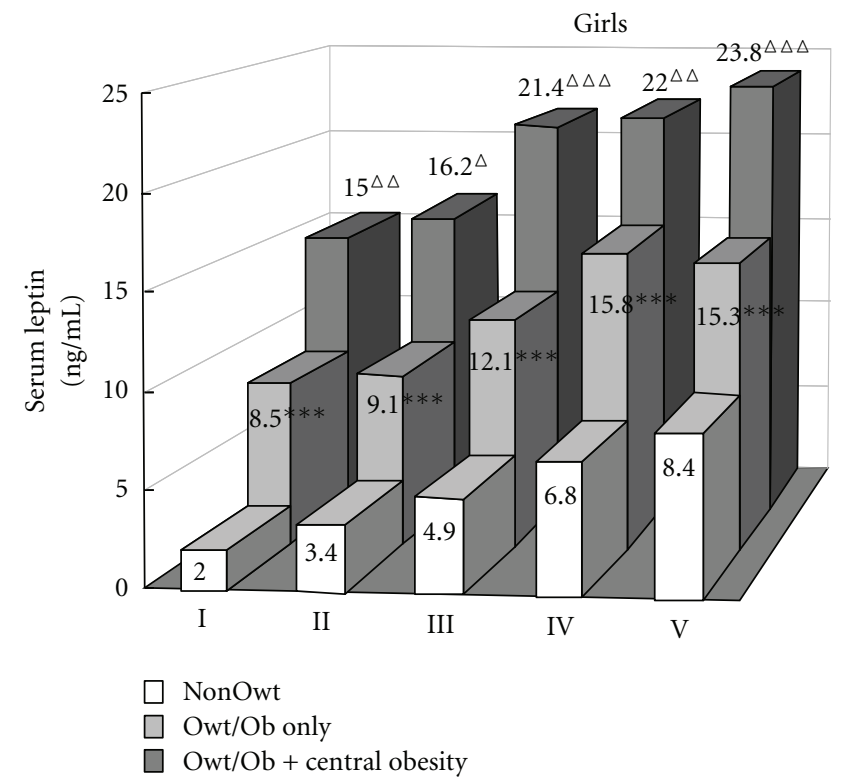

(a)

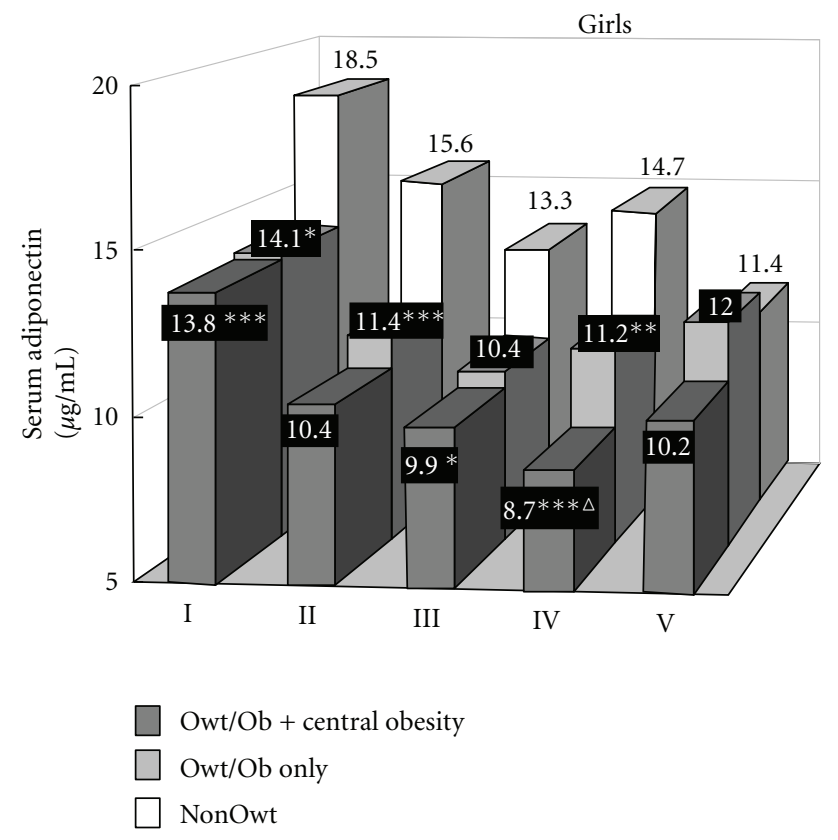

(c)

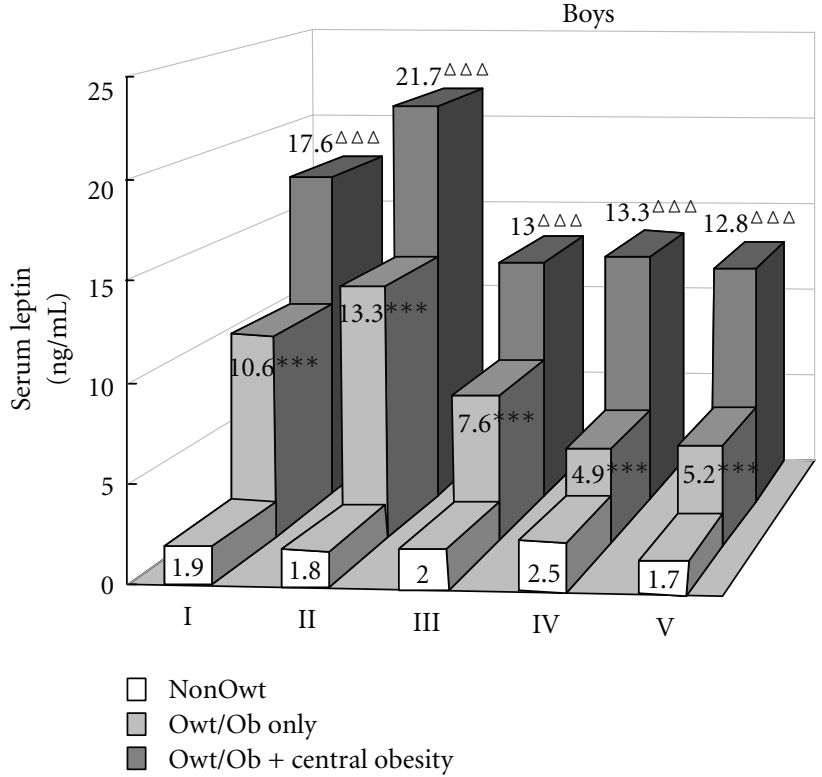

(b)

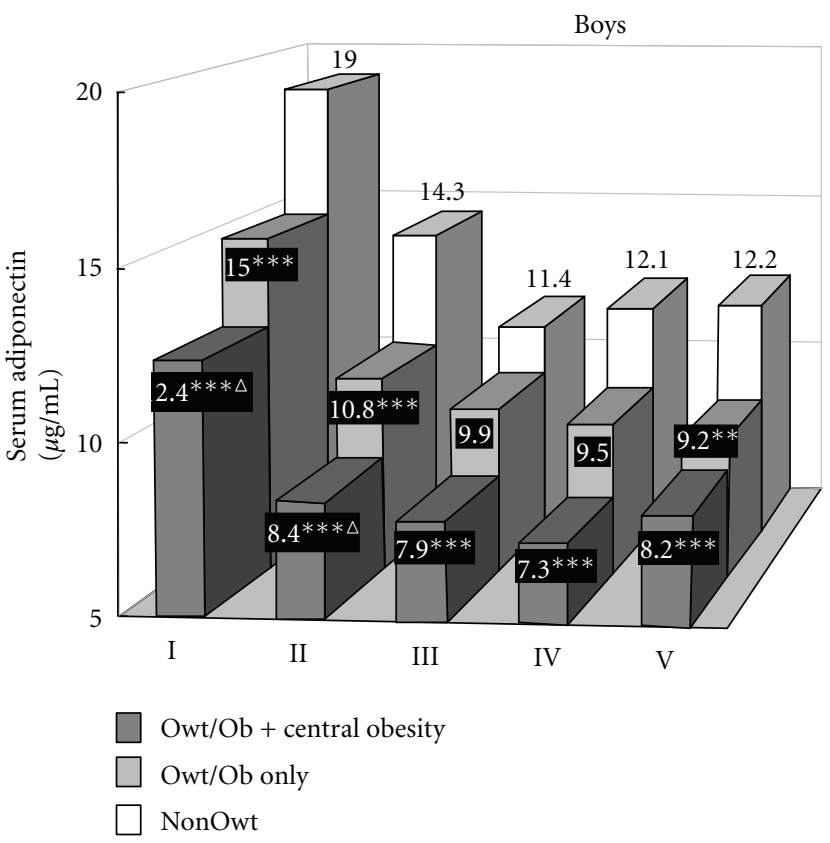

(d)

FIGURE 1: Leptin and adiponectin versus body size and central fat. Children were separated according to Tanner (puberty) and body size into non-overweight (NonOwt), overweight, and obese (Owt/Ob) \pm central obesity. Values are reported as leptin for girls (a), and boys (b), and mean adiponectin for girls (c) and boys (d). One way ANOVA is ${ }^{* *} P<.01,{ }^{* * *} P<.001$ versus NonOwt, ${ }^{\Delta} P<.01,{ }^{\Delta \Delta \Delta} P<.001 \mathrm{Owt} / \mathrm{Ob}$.

a gender-adjusted general linear model was used in analysis. Correlations were calculated using Pearson correlation coefficient. Significance was set at $P<.01$.

\section{Results}

3.1. Clinical Characteristics of Children and Adolescents. The baseline characteristics are summarized in Table 1. Subjects ranged from 6 to 18 years, distributed across all Tanner stages, with a mean age of $12.4 \pm 3.1$ years. Girls had a more advanced pubertal development than boys. Serum TC, LDL cholesterol, and insulin levels were not different between girls and boys, while leptin, adiponectin, and the ratio of leptin/adiponectin as well as body composition indices, HDL and glucose were different. Leptin and adiponectin correlated with multiple factors in both boys and girls, including BMI (used to evaluate overweight/obesity), waist circumference (the measure for central obesity), and factors 


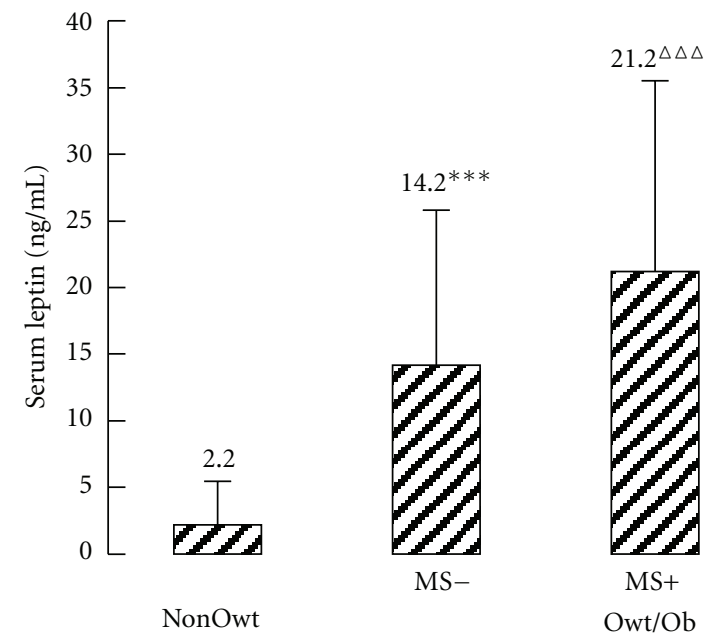

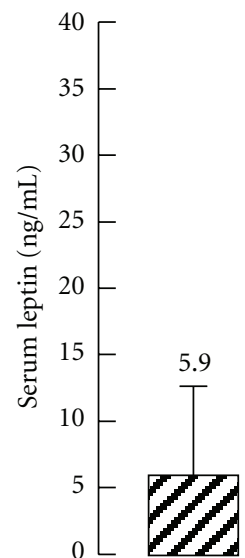

NonOwt

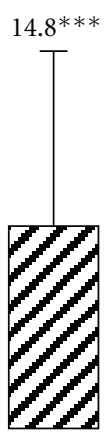

MS-

MS+

Owt/Ob

(a)

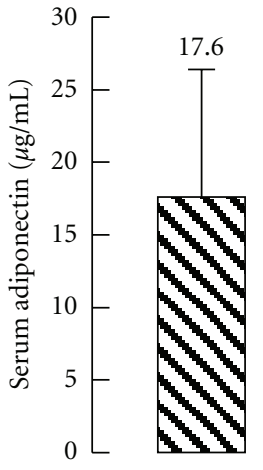

NonOwt

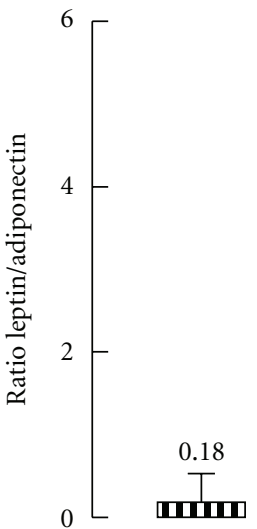

NonOwt

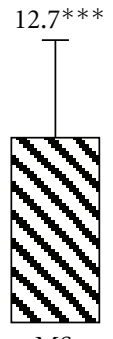

MS-

MS+

Owt/Ob

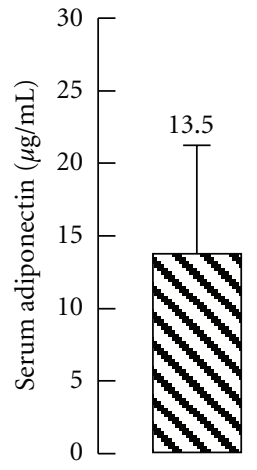

NonOwt

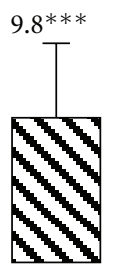

MS-

MS+

Owt/Ob

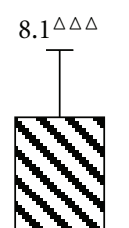$$
\mathrm{S}+
$$

(b)
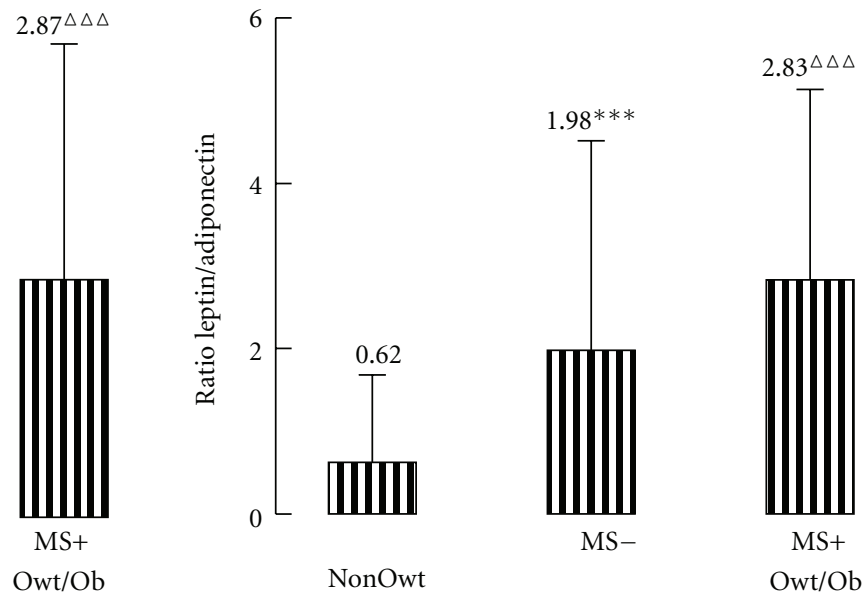

(c)

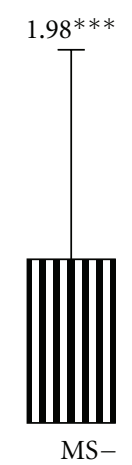

t/Ob

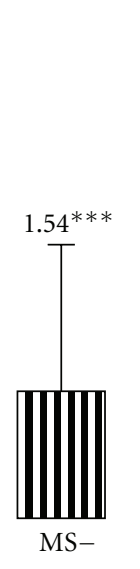

Owt/Ob

FIGURE 2: Leptin and adiponectin in overweight/obese children/adolescents \pm metabolic syndrome (MS) versus non-overweight (NonOwt) children. Children were separated according to early puberty (Tanner I + II) and late puberty (Tanner stage IV + V) and body size NonOwt and overweight/obese groups $(\mathrm{Owt} / \mathrm{Ob}) \pm \mathrm{MS}$. Mean \pm SD for leptin (a), adiponectin (b) and leptin/adiponectin (c) were analyzed using general linear model gender-adjusted $(P<.001)$, where ${ }^{* *} P<.01$, ${ }^{* * *} P<.001$ versus NonOwt and ${ }^{\Delta \Delta} P<.01, \Delta \Delta \Delta P<.001$ versus $\mathrm{OWt} / \mathrm{Ob}$ - MS. 
used in MS evaluation (blood pressure, triglyceride, HDL-C and glucose (data not shown)). Only leptin correlated with LDL cholesterol. Subjects were separated based on gender and BMI (normal weight (NonOwt) and overweight/obese (Owt/Ob). Leptin and adiponectin correlated with body weight parameters and metabolic indices including TG, HDL-C, and insulin (Table 2).

\subsection{Serum Adiponectin and Leptin Levels in Overweight/Obese} Subjects with Central Obesity. Children and adolescents were separated according to Tanner stage, gender and BMI (NonOwt or Owt/Ob only), and Owt/Ob with central obesity (Figure 1). In girls, leptin increased with Tanner stage, while adiponectin decreased. In boys, Tanner-related leptin changes were less pronounced, while adiponectin profile was similar to girls. Overall, leptin increased and adiponectin decreased in Owt/Ob in girls and boys $(P<$ $.001)$. Interestingly, central obesity had a pronounced effect on leptin, additional to overweight/obesity, in boys and girls (all Tanner stages) (Figures 1(a) and 1(b)). By contrast, central obesity impact on adiponectin was not additive to overweight/obesity alone, with little change (girls Tanner IV, boys Tanner I and II) (Figures 1(c) and 1(d)).

3.3. Adiponectin and Leptin Concentrations in Subjects with Overweight/Obesity with or without MS in Early and Late Puberty Stages. Children/adolescents grouped into early puberty (Tanner I/II) and late puberty (Tanner IV/V) were divided into NonOwt, Owt/Ob - MS, and Owt/Ob + MS. In early and late puberty, MS had additional effect on increasing leptin (Figure 2(a)) and decreasing adiponectin (Figure 2(b)) that was additive to overweight/obesity. Further, leptin/adiponectin ratio, which has been proposed as a biomarker, increased by $8.4-$ and 3.2 -fold in overweight/obesity, and by 15.8- and 4.5-fold with MS (Figure 2(c)). MS was associated with additive effects on waist circumference, blood pressure, TG, HDL-C, and glucose (parameters used to define MS), but also on other parameters (BMI, TC, LDL-C, and insulin) (data not shown). Even in early puberty ( $<10$ years), overweight/obesity influenced the adipokines leptin and adiponectin. Further, central obesity and MS were additive to overweight/obesity, and even at this young age, were associated with alterations in leptin, adiponectin and leptin-to-adiponectin ratio.

3.4. Metabolic Profiles in Upper Quintiles and Lower Quintiles of Leptin in Normal Weight and Overweight/Obese Children and Adolescents. While average leptin is significantly different between NonOwt and overweight/obese individuals, there is a substantial overlap between the distribution, such that some NonOwt have high leptin and some overweight/obese individuals have low leptin (comparable to NonOwt). This raises the question of whether NonOwt + high leptin presents a greater metabolic risk, and, conversely, whether obese + low leptin have better metabolic profile. This was evaluated in a gender- and puberty-specific manner. Upper quintile (top 20\% leptin, Q5) had mean leptin 10-15-fold greater than lower quintile group (bottom
20\% leptin, Q1) (Table 3). Interestingly, separation based on leptin does not discriminate for adiponectin with Q1 versus Q5 equivalent in NonOwt and overweight/obese. In NonOwt-Q5, although leptin is increased and within the overweight/obese range, BMI and WC remained normal. Finally, while Q5 leptin had no change in blood pressure, TC, LDL-C, or glucose (versus Q1), TG and insulin were different, suggesting that, separate from weight status, higher leptin may indicate additional metabolic risk.

3.5. Metabolic Profiles in Upper Quintiles and Lower Quintiles of Adiponectin in Normal Weight and Overweight/Obese Children and Adolescents. A similar analysis was conducted for adiponectin. While adiponectin distribution was shifted to lower values in overweight/obese subjects, there was substantial overlap, raising the questions: are NonOwt + low adiponectin at greater metabolic risk? are overweight/obese + high adiponectin more protected? In groups separated based on gender and puberty, upper quintile (Q5) and lower quintile (Q1) of adiponectin were compared (Table 4). Q5 NonOwt versus overweight/obese were similar regardless of weight status (same for Q1 groups), while Q5 was consistently 4-5-fold greater than Q1. Separation-based on adiponectin is non-discriminatory for leptin, with little difference in Q1 versus Q5, NonOwt and overweight/obese groups. Further, maximal \% difference between Q5 and Q1 for BMI and WC was never greater than $10 \%$. Adiponectin quintiles did not differentiate blood pressure, TC, LDL-C, or glucose, but Q1 adiponectin was generally associated with higher TG and insulin in NonOwt and overweight/obese children.

\section{Discussion}

In Asia, there has been an alarming increase in childhood/adolescent obesity, a major determinant of MS development $[1,2,17,18]$ a precursor to cardiovascular and Type 2 diabetes diseases [12, 24, 37], even in Chinese [3]. Adiponectin and leptin are correlated with MS in children and might be useful as a predictive marker of MS, although no consensus has been reached on this [12, 23-25, 27].

In the current study, we investigated adiponectin and leptin as diagnostic biomarkers in $>3500$ Beijing children/adolescents with varying weight and puberty. Leptin was increased and adiponectin decreased in overweight/obesity, with adiponectin decreasing with puberty (boys and girls) and leptin increasing with puberty (girls). The pubertal effect on these adipokines is consistent with German, American, and Japanese studies in children [26, 31], and has been suggested to be related to changes in sex hormones [19].

Leptin was strongly associated with central obesity, in contrast to adiponectin. Adiponectin, leptin, and leptin-toadiponectin ratio were strongly associated with MS, even at early puberty. These additive effects were consistent across puberty, in spite of adiponectin decreases (girls and boys) and leptin increases (girls only) with puberty. 
The most pertinent finding is the clustering of risk factors with increased leptin or decreased adiponectin which was present in NonOwt groups. NonOwt + high leptin or NonOwt + low adiponectin children manifested metabolic perturbations comparable to overweight/obese groups. This was evident in late, but also notably in early puberty, in boys and girls. Even in very young children, adipokines (leptin, adiponectin, leptin-to-adiponectin) might be valuable biomarkers for identifying NonOwt children at risk for later metabolic issues. However, one limitation of this study is the cross-sectional nature, and in the absence of a prospective longitudinal analysis, interpretation of the correlations can only be inferred.

Previous studies have demonstrated biomarker clustering in overweight/obese children, healthy children and both [12, 38-40], although these studies had only 300-600 subjects, in comparison to the large number of subjects in the present study, and did not necessarily cover the same age range. Interestingly, following lifestyle intervention in obese children, adiponectin proved to be the most significant predictor of improved metabolic profile, while changes in leptin were unrelated to positive metabolic outcomes [37]. Nonetheless, the clustering of multiple unfavourable biomarkers strongly supports the need for early intervention not only in childhood obesity, but also in normal weight subjects that manifest profiles at risk.

\section{Conclusion}

In summary, this large study of Chinese children and adolescents demonstrated leptin and adiponectin associations with central obesity and MS. Even in obesity, high leptin and low adiponectin predicts additional metabolic stress. Importantly, NonOwt with high leptin and low adiponectin predict an unfavourable metabolic profile regardless of gender/puberty. In future studies, followup of these NonOwt atrisk children may help to further demonstrate the usefulness of these biomarkers.

\section{Conflict of Interests}

The authors declare that they have no conflict of interests.

\section{Acknowledgments}

This study was supported by a Grant from the Canadian Institutes of Health Research to K. Cianflone (no77532), the Beijing Municipal Science and Technology Commission to J. Mi (H030930030031, D08050700320000), the National Natural Science Foundation of China to J. Mi (30872165 and 30911120198), FRSQ-NSFC Québec-China exchange program (K. Cianflone), doctoral scholarship from IRSC (M. N. Munkonda) and K.C. holds a Canada Research Chair in Adipose Tissue.

The authors appreciate the help of Mélanie Cianflone in manuscript preparation and technical assistance.

\section{References}

[1] J.-F. Fu, L. Liang, C.-C. Zou et al., "Prevalence of the metabolic syndrome in Zhejiang Chinese obese children and adolescents and the effect of metformin combined with lifestyle intervention," International Journal of Obesity, vol. 31, no. 1, pp. 15-22, 2007.

[2] C. Y. Ji and T. O. Cheng, "Epidemic increase in overweight and obesity in Chinese children from 1985 to 2005," International Journal of Cardiology, vol. 132, no. 1, pp. 1-10, 2009.

[3] B. M. Y. Cheung, N. M. S. Wat, S. Tam et al., "Components of the metabolic syndrome predictive of its development: a 6-year longitudinal study in Hong Kong Chinese," Clinical Endocrinology, vol. 68, no. 5, pp. 730-737, 2008.

[4] W. D.Johnson, J. J. M. Kroon, F. L. Greenway, C. Bouchard, D. Ryan, and P. T. Katzmarzyk, "Prevalence of risk factors for metabolic syndrome in adolescents: national health and nutrition examination survey (NHANES), 2001-2006," Archives of Pediatrics and Adolescent Medicine, vol. 163, no. 4, pp. 371377, 2009.

[5] Q. Zhuo, Z. Wang, P. Fu et al., "Comparison of adiponectin, leptin and leptin to adiponectin ratio as diagnostic marker for metabolic syndrome in older adults of Chinese major cities," Diabetes Research and Clinical Practice, vol. 84, no. 1, pp. 2733, 2009.

[6] C. B. Jasik and R. H. Lustig, "Adolescent obesity and puberty: the "perfect storm"," Annals of the New York Academy of Sciences, vol. 1135, pp. 265-279, 2008.

[7] B. Antuna-Puente, B. Feve, S. Fellahi, and J.-P. Bastard, "Adipokines: the missing link between insulin resistance and obesity," Diabetes and Metabolism, vol. 34, no. 1, pp. 2-11, 2008.

[8] A. M. G. Cali and S. Caprio, "Obesity in children and adolescents," Journal of Clinical Endocrinology and Metabolism, vol. 93, no. 11, pp. S31-S36, 2008.

[9] I. R. Madeira, C. N. M. Carvalho, F. M. Gazolla, L. W. Pinto, M. A. Borges, and M. A. N. Bordallo, "Impact of obesity on metabolic syndrome components and adipokines in prepubertal children," Jornal de Pediatria, vol. 85, no. 3, pp. 261-268, 2009.

[10] Y. Okamoto, S. Kihara, T. Funahashi, Y. Matsuzawa, and P. Libby, "Adiponectin: a key adipocytokine in metabolic syndrome," Clinical Science, vol. 110, no. 3, pp. 267-278, 2006.

[11] M. E. Trujillo and P. E. Scherer, "Adiponectin-journey from an adipocyte secretory protein to biomarker of the metabolic syndrome," Journal of Internal Medicine, vol. 257, no. 2, pp. 167-175, 2005.

[12] J. C. Winer, T. L. Zern, S. E. Taksali et al., "Adiponectin in childhood and adolescent obesity and its association with inflammatory markers and components of the metabolic syndrome," Journal of Clinical Endocrinology and Metabolism, vol. 91, no. 11, pp. 4415-4423, 2006.

[13] X. Fang and G. Sweeney, "Mechanisms regulating energy metabolism by adiponectin in obesity and diabetes," Biochemical Society Transactions, vol. 34, no. 5, pp. 798-801, 2006.

[14] M. Shargorodsky, M. Boaz, Y. Goldberg et al., "Adiponectin and vascular properties in obese patients: is it a novel biomarker of early atherosclerosis?" International Journal of Obesity, vol. 3, no. 5, pp. 553-558, 2009.

[15] E. Jéquier, "Leptin signaling, adiposity, and energy balance. Lipids and Insulin Resistance: the Role of Fatty Acid Metabolism and Fuel Partitioning," Annals of the New York Academy of Sciences, vol. 967, pp. 379-388, 2002. 
[16] O. Moran and M. Phillip, "Leptin: obesity, diabetes and other peripheral effects-a review," Pediatric Diabetes, vol. 4, no. 2 , pp. 101-109, 2003.

[17] C.-C. Liao, T.-C. Su, K.-L. Chien et al., "Elevated blood pressure, obesity, and hyperlipidemia," Journal of Pediatrics, vol. 155, no. 1, pp. 79-83, 2009.

[18] Y. Wu, "Overweight and obesity in China," British Medical Journal, vol. 333, no. 7564, pp. 362-363, 2006.

[19] P. B. Kaplowitz, "Link between body fat and the timing of puberty," Pediatrics, vol. 121, supplement 3, pp. S208-S217, 2008.

[20] L. A. Moreno, C. Tomás, M. González-Gross, G. Bueno, J. M. Pérez-González, and M. Bueno, "Micro-environmental and socio-demographic determinants of childhood obesity," International Journal of Obesity, vol. 28, supplement 3, pp. S16-S20, 2004.

[21] A. Körner, S. Blüher, T. Kapellen et al., "Obesity in childhood and adolescence: a review in the interface between adipocyte physiology and clinical challenges," Hormones, vol. 4, no. 4, pp. 189-199, 2005.

[22] F. Eyzaguirre and V. Mericq, "Insulin resistance markers in children," Hormone Research, vol. 71, no. 2, pp. 65-74, 2009.

[23] L. A. Moreno, I. Pineda, G. Rodríguez et al., "Leptin and metabolic syndrome in obese and non-obese children," Hormone and Metabolic Research, vol. 34, no. 7, pp. 394-399, 2002.

[24] G. Q. Shaibi, M. L. Cruz, M. J. Weigensberg et al., "Adiponectin independently predicts metabolic syndrome in overweight Latino youth," Journal of Clinical Endocrinology and Metabolism, vol. 92, no. 5, pp. 1809-1813, 2007.

[25] J. Steinberger, L. Steffen, D. R. Jacobs Jr., A. Moran, C.P. Hong, and A. R. Sinaiko, "Relation of leptin to insulin resistance syndrome in children," Obesity Research, vol. 11, no. 9, pp. 1124-1130, 2003.

[26] R. Nishimura, H. Sano, T. Matsudaira et al., "Changes in body mass index, leptin and adiponectin in Japanese children during a three-year follow-up period: a population-based cohort study," Cardiovascular Diabetology, vol. 8, article 30, 2009.

[27] S. Zhang, X. Liu, W. J. Brickman et al., "Association of plasma leptin concentrations with adiposity measurements in rural Chinese adolescents," Journal of Clinical Endocrinology and Metabolism, vol. 94, no. 9, pp. 3497-3504, 2009.

[28] N. Oda, S. Imamura, T. Fujita et al., "The ratio of leptin to adiponectin can be used as an index of insulin resistance," Metabolism: Clinical and Experimental, vol. 57, no. 2, pp. 268 273, 2008.

[29] N. Satoh, M. Naruse, T. Usui et al., "Leptin-to-adiponectin ratio as a potential atherogenic index in obese type 2 diabetic patients," Diabetes Care, vol. 27, no. 10, pp. 2488-2490, 2004.

[30] O. A. Mojiminiyi, N. A. Abdella, M. Al Arouj, and A. Ben Nakhi, "Adiponectin, insulin resistance and clinical expression of the metabolic syndrome in patients with Type 2 diabetes," International Journal of Obesity, vol. 31, no. 2, pp. 213-220, 2007.

[31] C. Koebnick, G. Q. Shaibi, L. A. Kelly et al., "Leptin-toadiponectin ratio as independent predictor of insulin sensitivity during growth in overweight Hispanic youth," Journal of Endocrinological Investigation, vol. 30, no. 7, pp. RC13-RC16, 2007.

[32] M. Li, A. Fisette, X.-Y. Zhao, J.-Y. Deng, J. Mi, and K. Cianflone, "Serum resistin correlates with central obesity but weakly with insulin resistance in Chinese children and adolescents," International Journal of Obesity, vol. 33, no. 4, pp. 424-439, 2009.

[33] T. J. Cole, M. C. Bellizzi, K. M. Flegal, and W. H. Dietz, "Establishing a standard definition for child overweight and obesity worldwide: international survey," British Medical Journal, vol. 320, no. 7244, pp. 1240-1243, 2000.

[34] L. Ming, W. Congyuan, S. Ailing, and Z. Kui, "Development and preliminary application of enzyme-linked immunosorbent assay for human net insulin in serum," The Journal of Clinical Endocrinology \& Metabolism, vol. 13, pp. 214-217, 1997.

[35] K. Zhang, M. Li, and C. Y. Wu, "reparation of antibodies against human leptin and evelopement on new generation ELISA for human serum leptin," Hong Kong Medical Journal, vol. 7, supplement 2, pp. 139-139, 2001.

[36] N. J. Wan, J. Mi, T. Y. Wang et al., "Metabolic syndrome in overweight and obese school children in Beijing," Zhonghua Er Ke Za Zhi, vol. 6, pp. 417-421, 2007.

[37] V. M. Cambuli, M. C. Musiu, M. Incani et al., "Assessment of adiponectin and leptin as biomarkers of positive metabolic outcomes after lifestyle intervention in overweight and obese children," Journal of Clinical Endocrinology and Metabolism, vol. 93, no. 8, pp. 3051-3057, 2008.

[38] A. Kettaneh, B. Heude, J.-M. Oppert et al., "Serum adiponectin is related to plasma high-density lipoprotein cholesterol but not to plasma insulin-concentration in healthy children: the FLVS II study," Metabolism: Clinical and Experimental, vol. 55, no. 9, pp. 1171-1176, 2006.

[39] G. Nagel, K. Rapp, M. Wabitsch et al., "Prevalence and cluster of cardiometabolic biomarkers in overweight and obese schoolchildren: results from a large survey in Southwest Germany," Clinical Chemistry, vol. 54, no. 2, pp. 317-325, 2008.

[40] M. Yoshinaga, K. Sameshima, Y. Tanaka et al., "Adipokines and the prediction of the accumulation of cardiovascular risk factors or the presence of metabolic syndrome in elementary school children," Circulation Journal, vol. 72, no. 11, pp. 18741878, 2008. 


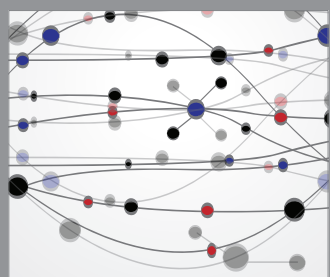

The Scientific World Journal
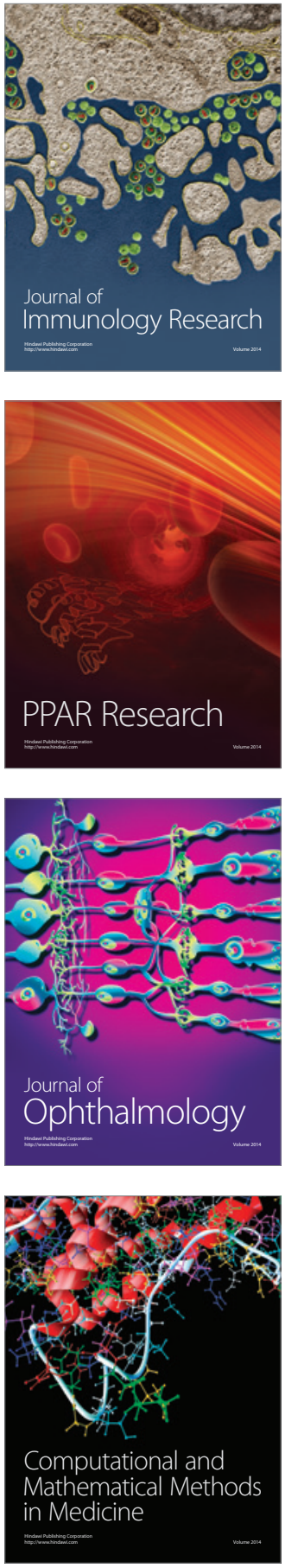

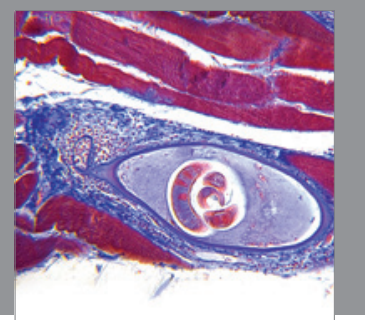

Gastroenterology

Research and Practice
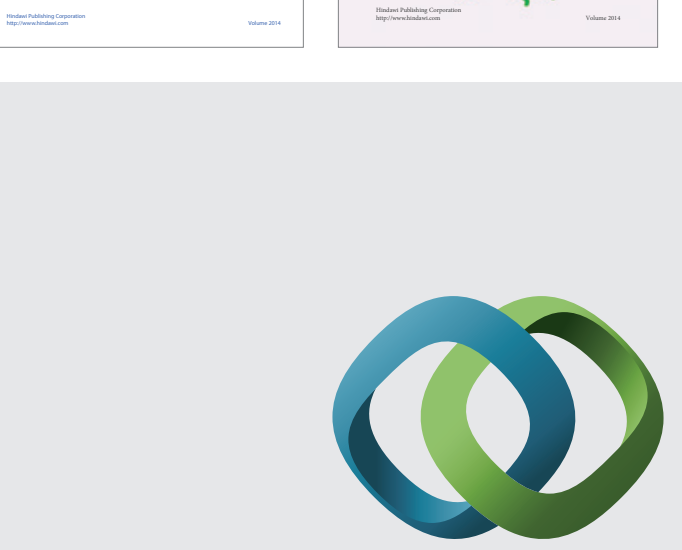

\section{Hindawi}

Submit your manuscripts at

http://www.hindawi.com
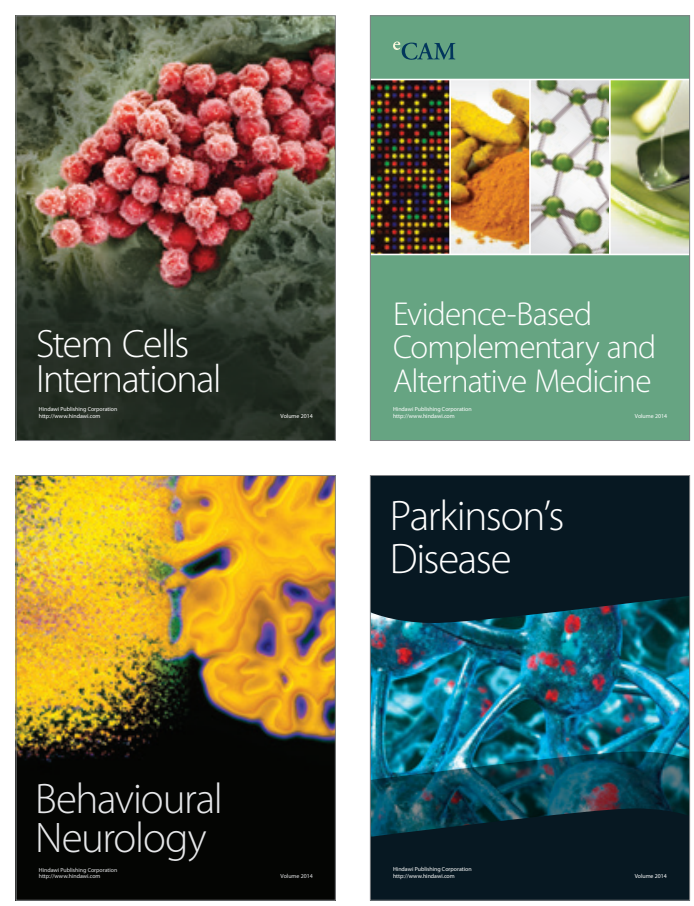

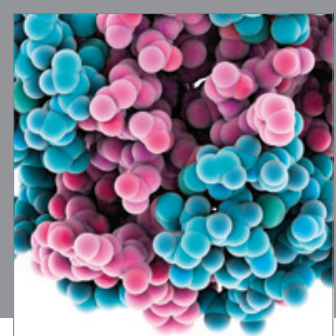

Journal of
Diabetes Research

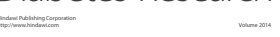

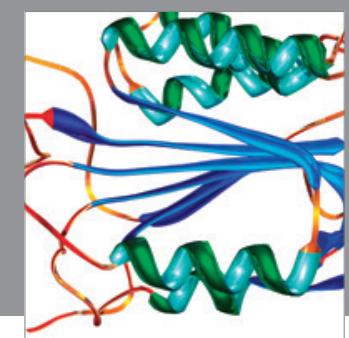

Disease Markers
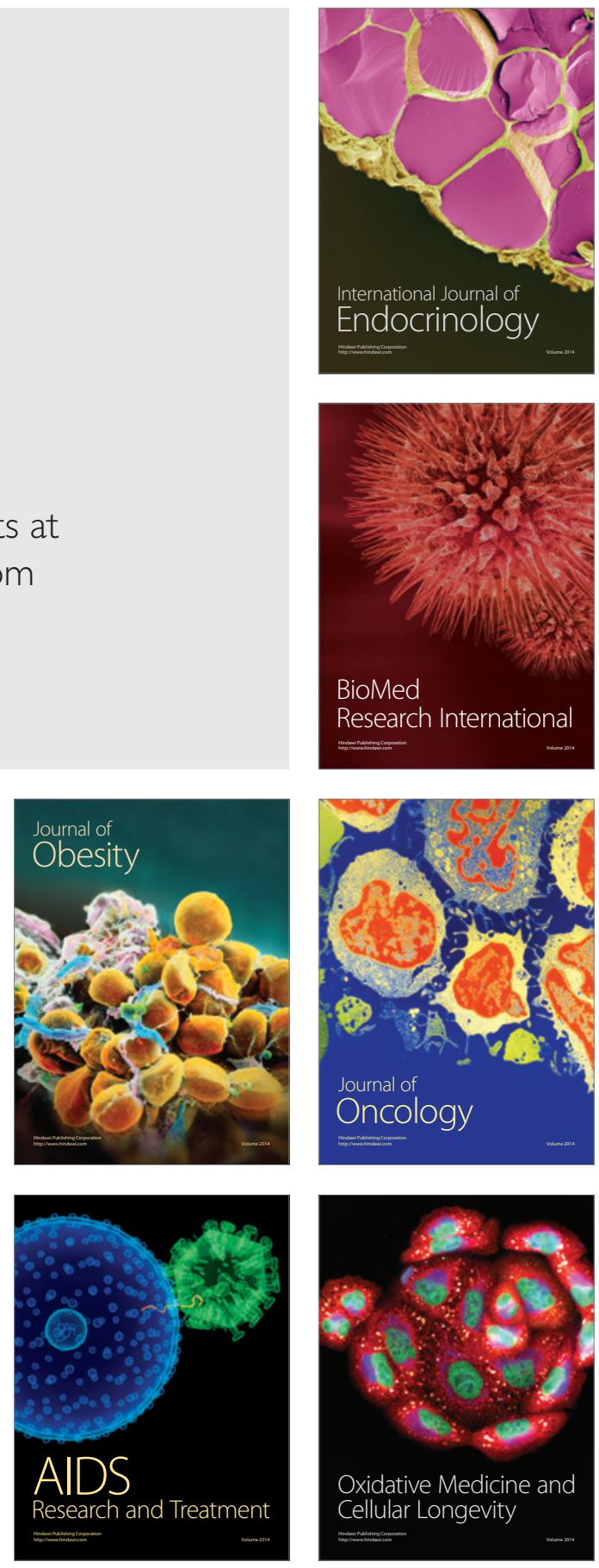